\title{
A CIRCUIT-PRESERVING MAPPING FROM MULTILEVEL TO BOOLEAN DYNAMICS
}

\author{
ADRIEN FAURÉ AND SHIZUO KAJI
}

\begin{abstract}
Many discrete models of biological networks rely exclusively on Boolean variables and many tools and theorems are available for analysis of strictly Boolean models. However, multilevel variables are often required to account for threshold effects, in which knowledge of the Boolean case does not generalise straightforwardly. This motivated the development of conversion methods for multilevel to Boolean models. In particular, Van Ham's method has been shown to yield a one-to-one, neighbour and regulation preserving dynamics, making it the de facto standard approach to the problem. However, Van Ham's method has several drawbacks: most notably, it introduces vast regions of "non-admissible" states that have no counterpart in the multilevel, original model. This raises special difficulties for the analysis of interaction between variables and circuit functionality, which is believed to be central to the understanding of dynamic properties of logical models. Here, we propose a new multilevel to Boolean conversion method, with software implementation. Contrary to Van Ham's, our method doesn't yield a one-to-one transposition of multilevel trajectories; however, it maps each and every Boolean state to a specific multilevel state, thus getting rid of the non-admissible regions and, at the expense of (apparently) more complicated, "parallel" trajectories. One of the prominent features of our method is that it preserves dynamics and interaction of variables in a certain manner. As a demonstration of the usability of our method, we apply it to construct a new Boolean counter-example to the well-known conjecture that a local negative circuit is necessary to generate sustained oscillations. This result illustrates the general relevance of our method for the study of multilevel logical models.
\end{abstract}

\section{BACKGROUND}

Boolean models have proved very useful in the analysis of various networks in biology. However, it is often convenient to introduce multilevel variables to account for multiple threshold effects. We are often faced with choices between using Boolean variables or multilevel variables. This can be crucial since theoretical results are sometimes proved only for Boolean or multilevel networks. A particular example of this situation is in René Thomas' conjecture that a local negative circuit is necessary to produce sustained (asynchronous) oscillations. This paper stems from the simple idea that a Boolean counter-example to that conjecture could be found by transposing a multilevel counter-example found earlier by Richard and Comet. However, we believe the method developed in this paper, together with a handy script which implements it, is widely applicable to other theoretical studies which involves discrete networks. We also find the notion of asymptotic evolution function defined in this paper sheds light on the understanding of relation between the state transition graph and the interaction graph.

1.1. Introduction. Introduced in the 1960s-70s to model biological regulatory networks, the logical (discrete) formalism has gained increasing popularity, with recent applications as diverse as drosophila development, cell cycle control, or immunology (see [1] for a survey). While many of these models rely exclusively on Boolean variables, it is often useful to introduce multilevel variables to account for more refined behaviour. However, many tools and theoretical results are restricted to the Boolean case (see e.g. [2, 3, 4]) This situation motivated the development of methods to convert multilevel models to Boolean ones [5, 6]. A simple idea for such a conversion was introduced by Van Ham [6], and this method has been shown to be essentially the only one that could provide a "one-to-one, neighbour and regulation preserving map" [7]. One problem with the conversion is that

2010 Mathematics Subject Classification. Primary 68R05; Secondary 92D99.

Key words and phrases. Discrete dynamical system, Automata network, Boolean network, Regulatory network, Genetic regulation, Thomas' conjecture.

The authors contributed equally to this work. 
the resulting Boolean model is defined only on a sub-region of the whole Boolean state space, called the admissible region, and how to extend the model outside that region is not trivial. This leads to potential problems with analytical tools designed to deal with the whole state space, as a property that is true in the restricted domain may be false on the whole state space, and vice versa. The primary goal of the present paper is to address this issue by introducing an extension of Van Ham's method. More precisely, we introduce a new method for multilevel to Boolean model conversion which extends the domain of Van Ham's model to the whole state space while preserving edge functionality and, therefore, local circuits. Our mapping yields a state transition graph with "parallel" trajectories that contains the one obtained by Van Ham's mapping as a sub-graph in such a way that attractors of the dynamics are preserved.

We apply our method to investigate a particular class of theoretical results that connect the asynchronous behaviour of a model to the presence of regulatory circuits in the interaction graph. In the early 1980s, R. Thomas conjectured that the presence of a positive circuit (i.e. a circuit where each component directly or indirectly has a positive effect upon itself) in the interaction graph is a necessary condition for multi-stability, and a negative circuit (where each component has a negative effect upon itself) is necessary for sustained oscillations [8]. One particular formulation of the conjecture focuses on local or "type-1" circuits [9], i.e. circuits whose arcs are all functional in the same single point of the system's state transition graph - as opposed to global circuits whose arcs may be functional anywhere. While the conjecture holds for positive circuits both at the global and local levels, and for multilevel as well as Boolean models [10, 11], in the negative case the conjecture could only be proved true at the global level [10]. At the local level, a counter-example has first been published for multilevel models [12], while the Boolean case remained open [9] until a Boolean counter-example was eventually discovered [13], showing that contrary to expectations, a local negative circuit was not necessary to generate sustained oscillations. Interestingly, the approaches taken by P. Ruet and A. Richard are rather different, and their counter-examples have little in common. Applying our method to the Richard-Comet multilevel counter-example, we obtain a new Boolean counter-example to the conjecture that a local negative circuit is necessary to produce sustained oscillations.

\subsection{Definitions.}

1.2.1. Evolution function and State transition graph. We work within the generalised logical framework introduced by René Thomas and collaborators [14]; see Abou-Jaoudé et al. [1] for a recent review. Here, we introduce the notation we use throughout this paper. Fix positive integers $n$ and $m_{i}(1 \leq i \leq n)$. Consider a system consisting of mutually interacting $n$ genes, indexed by the set $I=\{1,2, \ldots, n\}$. Each gene $a_{i}$ takes expression levels in the integer interval $\left\{0,1, \ldots, m_{i}\right\}$. The state of the system evolves depending on the current state. This leads to a discrete dynamical system represented by a evolution function over $M$

$$
f=\left(f_{1}, f_{2}, \ldots, f_{n}\right): M \rightarrow M,
$$

where $M=\left\{\left(x_{1}, \ldots, x_{n}\right) \mid x_{i} \in\left\{0,1, \ldots, m_{i}\right\}\right\}$. As a special case when $m_{i}=1$ for all $i \in I$, we denote $M=\mathbb{B}^{n}$ with $\mathbb{B}=\{0,1\}$ and call the system Boolean. A basic question asks what we can tell about the asymptotic global behaviour of the dynamics, which is encoded in the state transition graph, from local data of $f$, which are encoded in the partial derivatives of $f$ or the interaction graph.

The evolution of the whole system can be formally modelled by a certain kind of directed graph on $M$. We equip $M$ with the usual metric $d\left(x, x^{\prime}\right)=\sum_{i=1}^{n}\left|x_{i}-x_{i}^{\prime}\right|$ for $x, x^{\prime} \in M$. Denote by $e_{1}=$ $(1,0,0, \ldots), e_{2}=(0,1,0,0, \ldots)$, etc. the coordinate vectors of $M$. A grid graph $\Gamma$ over $M$ is a graph with the vertex set $M$ satisfying that

- each directed edge connects a pair of vertices of distance one

- at each vertex $x$ there are no two parallel outward edges; that is, $x-e_{j} \leftarrow x \rightarrow x+e_{j}$ is not allowed.

The state of the whole system is represented by the levels of genes, and corresponds to a vertex in $\Gamma$. At each time step, the state evolves to one of its neighbouring vertices connected by an arrow in the 
following way. To an evolution function over $M$, we associate a grid graph $\Gamma(f)$ over $M$ called the (asynchronous) state transition graph with the edge set

$$
\left\{\left(x_{1}, x_{2}, \ldots, x_{j}, \ldots, x_{n}\right) \rightarrow\left(x_{1}, x_{2}, \ldots, x_{j}+\delta, \ldots, x_{n}\right), \delta=\left\{\begin{array}{ll}
-1 & \left(f_{j}(x)<x_{j}\right) \\
+1 & \left(f_{j}(x)>x_{j}\right)
\end{array}\right\} .\right.
$$

Note that here we follow the standard convention that transition of states is unitary (see [12, §4]) so that the existence of an edge $x \rightarrow x^{\prime}$ implies $d\left(x, x^{\prime}\right)=1$; that is, at each step the level of a single gene changes at most by one.

Asymptotic behaviour of the evolution of a system can be captured in a graph theoretical entity of the state transition graph. An attractor is a terminal strongly connected sub-graph of $\Gamma$; that is, any two elements of it are connected by a path and there is no edge from its elements to one in the complement. An attractor consisting of a single vertex is called a stable state, otherwise it is called a cyclic attractor. Intuitively, attractors are domains in $\Gamma$ in which the system eventually resides; there is no way to escape once the system arrives in it, but each state in the domain can be visited after arbitrarily many steps.

1.2.2. Interaction graph and circuit functionality. A common practice in analysing interactions among genes in a network is to encode it in the form of a labelled directed graph called the interaction graph, where interaction is measured by the partial derivatives of the evolution function $f=\left(f_{1}, f_{2}, \ldots, f_{n}\right)$ : $M \rightarrow M$.

The forward partial derivative of $f_{i}$ along the $j$-th coordinate at $x=\left(x_{1}, \ldots, x_{n}\right)$ with $x_{j}<m_{j}$ is defined by

$$
\partial_{j}^{+} f_{i}(x)=f_{i}\left(x_{1}, \ldots, x_{j}+1, \ldots, x_{n}\right)-f_{i}\left(x_{1}, \ldots, x_{j}, \ldots, x_{n}\right)=f_{i}\left(x+e_{j}\right)-f_{i}(x) .
$$

The backward partial derivative along the $j$-th coordinate at $x$ with $x_{j}>0$ is defined similarly by

$$
\partial_{j}^{-} f_{i}(x)=f_{j}\left(x_{1}, \ldots, x_{j}, \ldots, x_{n}\right)-f_{j}\left(x_{1}, \ldots, x_{j}-1, \ldots, x_{n}\right)=f_{i}(x)-f_{i}\left(x-e_{j}\right) .
$$

Partial derivatives $\partial_{j}^{+} f_{i}(x)$ and $\partial_{j}^{-} f_{i}(x)$ are non-trivial when the $i$-th gene's target value changes along the change of the $j$-th gene. They encode the dependence between genes locally at the state $x \in M$.

Remark 1. For a Boolean network, only one of the forward or the backward partial derivative exists at each $x$, so we just put them together to define the ordinary partial derivative denoted by $\partial_{j}$. On the other hand, in multilevel case, we have both the forward and the backward partial derivatives at some $x$. It is important to consider both of them (c.f. [12, Definition 8]).

Definition 1. The (local) interaction graph $G f(x)$ of $f$ at $x$ is a graph over the vertex set $I$ such that there exists an edge from $j$ to $i$

- with label " + " if $\partial_{j}^{+} f_{i}(x)>0$ or $\partial_{j}^{-} f_{i}(x)>0$

- with label "-"“if $\partial_{j}^{+} f_{i}(x)<0$ or $\partial_{j}^{-} f_{i}(x)<0$.

Note that we can have both positive and negative edges from $j$ to $i$ at the same time. We define the global interaction graph $G f(M)$ as the union of edges of $G f(x)$ for all $x \in M$.

Definition 2 ([9, Definition 1, Proposition 1]).

- A cycle $C$ in $G f(M)$ is called a positive (resp. negative) circuit if it contains an even (resp. odd) number of negative edges.

- A circuit $C$ is said to be type-1 functional if $C \subset G f(x)$ for some $x$.

As in the continuous case, a function $f$ is recovered up to constant by its partial derivatives: For two evolution functions $f, g: M \rightarrow M$ satisfying $\partial_{j}^{+} f_{i}=\partial_{j}^{+} g_{i}$ for all $i, j \in I$ (or $\partial_{j}^{-} f_{i}=\partial_{j}^{-} g_{i}$ for all $i, j \in I$ ), the difference $f_{i}(x)-g_{i}(x)$ is constant for any $i \in I$. In particular, two distinct Boolean evolution functions have the same partial derivatives if and only if they are constant and do not coincide at any point. This means the partial derivatives have almost all the information of the network However, the next example shows that the partial derivatives are not enough to determine the asymptotic behaviour of the dynamics. 
Example 1. Consider the the Boolean evolution functions defined by

$$
f\left(x_{1}, x_{2}\right)=\left\{\begin{array}{ll}
(0,0) & \left(\left(x_{1}, x_{2}\right)=(1,0)\right) \\
(1,0) & (\text { otherwise })
\end{array} \quad g\left(x_{1}, x_{2}\right)= \begin{cases}(0,1) & \left(\left(x_{1}, x_{2}\right)=(1,0)\right) \\
(1,1) & (\text { otherwise })\end{cases}\right.
$$

Since they differ by a constant, their partial derivatives agree. There exists the unique cyclic attractor $(0,0) \leftrightarrow(1,0)$ in $\Gamma(f)$, whereas there exists the unique stable state $(1,1)$ in $\Gamma(g)$.

\section{Methods}

2.1. Asymptotic evolution function. The correspondence between evolution functions and state transition graphs is not bijective. In fact, as discussed by Streck et al. [15], for a given (multilevel) grid graph $\Gamma$, there are multiple evolution functions which have $\Gamma$ as their state transition graphs. To have a bijective correspondence between the two representations of the system, we restrict ourselves to a certain class of evolution functions. There are two major conventions:

(1) We say $f$ is stepwise or unitary if $\left|f_{i}\left(x_{1}, \ldots, x_{n}\right)-x_{i}\right| \leq 1$ for all $i \in I$ and $x \in M$.

(2) We say $f$ is asymptotic if $f_{i}\left(x_{1}, \ldots, x_{i}, \ldots, x_{n}\right) \in\left\{0, x_{i}, m_{i}\right\}$ for all $i \in I$ and $x \in M$.

In both cases, $f_{i}$ encodes only the sign of $f_{i}(x)-x_{i}$.

For any evolution function, there exists a unique asymptotic and a unique stepwise evolution functions having the same state transition graph.

Proposition 1. For any evolution function $f$, define

$$
\bar{f}_{i}(x)=\left\{\begin{array}{ll}
m_{i} & \left(f_{i}(x)>x_{i}\right) \\
x_{i} & \left(f_{i}(x)=x_{i}\right) \\
0 & \left(f_{i}(x)<x_{i}\right)
\end{array} \quad \hat{f}_{i}(x)= \begin{cases}x_{i}+1 & \left(f_{i}(x)>x_{i}\right) \\
x_{i} & \left(f_{i}(x)=x_{i}\right) \\
x_{i}-1 & \left(f_{i}(x)<x_{i}\right)\end{cases}\right.
$$

Then, $\bar{f}$ is asymptotic and $\hat{f}$ is stepwise with $\Gamma(f)=\Gamma(\bar{f})=\Gamma(\hat{f})$.

Similarly, for any grid graph $\Gamma$, there exists a unique asymptotic function $\bar{f}^{\Gamma}$ and a unique stepwise function $\hat{f}^{\Gamma}$ such that $\Gamma=\Gamma\left(\bar{f}^{\Gamma}\right)=\Gamma\left(\hat{f}^{\Gamma}\right)$.

Proof. We see how to define $\bar{f}^{\Gamma}$. For a vertex $x \in \Gamma$ and $i \in I$, we have only one of the three possibilities:

- $x \rightarrow x-e_{i}$

- $x \rightarrow x+e_{i}$

- there is no edge from $x$ in the direction of $e_{i}$.

We define an asymptotic evolution function $\bar{f}^{\Gamma}$ by setting $f_{i}^{\Gamma}(x)=0, m, x_{i}$ accordingly.

If we are interested in the evolution of a system, which is encoded in the state transition graph, we can restrict ourselves to either the class of stepwise evolution functions or the class of asymptotic evolution functions. Our choice in this paper is to restrict ourselves to the latter, and we identify an asymptotic evolution function with its state transition graph and vice versa. In the rest of the paper, we assume functions are asymptotic unless otherwise stated and denoted just by $f$ without a bar over it.

There is a little difference in the interaction graph when we consider the stepwise case instead of the asymptotic case. When $i \neq j, \partial_{i}^{+} f_{j}(x)$ and $\partial_{i}^{+} \hat{f}_{j}(x)$ have the same sign, and same is true for the backward partial derivatives. However, when $i=j$, we have the following difference.

Example 2. Consider the asymptotic evolution function $f_{1}(0)=2, f_{1}(1)=2, f_{1}(2)=2$ over $M=$ $\{0,1,2\}$. The corresponding stepwise evolution function is $\hat{f}_{1}(0)=1, \hat{f}_{1}(1)=2, \hat{f}_{1}(2)=2$. At $x=1$, there is no edges in the interaction graph of $f$ while there is a positive self-loop in the one of $\hat{f}$. On the other hand, consider the asymptotic evolution function $f_{1}(0)=2, f_{1}(1)=1, f_{1}(2)=0$. At $x=1$, there is a negative self-loop in the interaction graph of $f$, whereas there is no arrow in the one of the corresponding stepwise evolution function $\hat{f}_{1}(0)=1, \hat{f}_{1}(1)=1, \hat{f}_{1}(2)=1$. 
In short, the interaction graphs of an asymptotic function and its corresponding stepwise function are the same only up to self-loops.

A function which is neither asymptotic nor stepwise has in general more non-trivial partial derivatives than the asymptotic and the stepwise functions sharing the same state transition graph given in Proposition 1. (See [15] for a detailed discussion. The stepwise function in our paper can be seen as a special case of the canonical function defined there.)

Example 3. An asymptotic function $f:\{0,1,2\}^{2} \rightarrow\{0,1,2\}^{2}$ defined by $\left(f_{1}, f_{2}\right)\left(x_{1}, x_{2}\right)=(2,2)$ have the same state transition graph with

$$
\left(g_{1}, g_{2}\right)\left(x_{1}, x_{2}\right)=\left\{\begin{array}{ll}
(1,2) & \left(x_{1}, x_{2}\right)=(0,0) \\
(2,2) & (\text { otherwise })
\end{array} .\right.
$$

However, $\partial_{2} f_{1}(0,0)=0$ and $\partial_{2} g_{1}(0,0)=1$. This means, there is a positive arrow $x_{2} \rightarrow x_{1}$ in $\operatorname{Gg}(0,0)$ while there is no arrow in $G f(0,0)$.

2.2. A mapping from multilevel to Boolean networks. Fix the set of states $M$ and a natural number $l$. We consider mappings from the $\operatorname{set} \operatorname{Evo}(M)$ of asymptotic evolution functions on $M$ to the set $\operatorname{Evo}\left(\mathbb{B}^{l}\right)$ of $l$-dimensional Boolean evolution functions. Mappings between grid graphs are obtained from them by the correspondence given in Proposition 17. Following [7], we introduce two preferable properties of such mappings.

Definition 3. A mapping $\Psi: \operatorname{Evo}(M) \rightarrow \operatorname{Evo}\left(\mathbb{B}^{l}\right)$ is said to be

- neighbour-preserving if there exists a map $b: M \rightarrow \mathbb{B}^{l}$ and $\psi: \mathbb{B}^{l} \rightarrow M$ such that $\psi \circ b$ is the identity on $M$ and $b$ and $\psi$ induce graph homomorphisms $\tilde{b}: \Gamma(f) \rightarrow \Gamma(\Psi(f))$ and $\tilde{\psi}: \Gamma(\Psi(f)) \rightarrow \Gamma(f)$ for any $f \in \operatorname{Evo}(M)$.

- globally regulation-preserving if $G \Psi(f)\left(\mathbb{B}^{l}\right) \neq G \Psi\left(f^{\prime}\right)\left(\mathbb{B}^{l}\right)$ for any $f, f^{\prime} \in \operatorname{Evo}(M)$ with $G f(M) \neq G f^{\prime}(M)$.

- locally regulation-preserving if there exists a map $b: M \rightarrow \mathbb{B}^{l}$ such that $G \Psi(f)(b(y)) \neq$ $G \Psi\left(f^{\prime}\right)(b(y))$ for any $y \in M$ and any $f, f^{\prime} \in \operatorname{Evo}(M)$ with $G f(y) \neq G f^{\prime}(y)$.

These properties are practically useful. For a neighbour-preserving mapping, the two maps $b$ and $\psi$ give correspondence between the multilevel states and the Boolean states in such a way that the state transition graph of any multilevel model is embedded in that of a Boolean model. With a regulationpreserving mapping, one can recover the interaction graph of a multilevel network from the corresponding Boolean one.

A naive idea to convert an evolution function $f: M \rightarrow M$ to a Boolean one is to use an embedding (one-to-one map) $b: M \rightarrow \mathbb{B}^{l}$ of the set of multilevel states to a higher dimensional set of Boolean states. Then, the conjugate of $f$ with respect to $b$ is defined as

$$
f_{b}(x):=b \circ f \circ b^{-1}(x),
$$

which is defined only on $\operatorname{Im}(b) \subset \mathbb{B}^{l}$, the image of $b$. The domain $\operatorname{Im}(b)$ is called the admissible region for $f_{b}$. The state transition graph $\Gamma\left(f_{b}\right)$ in this case is defined to be the full sub-graph on $\operatorname{Im}(b)$ of the one defined by Eq. (1). Van Ham [6] proposed one particular embedding $b_{0}: M \rightarrow \mathbb{B}^{m_{1}+m_{2}+\cdots+m_{n}}$ which is defined as the direct product of

$$
\left(b_{0}\right)_{i}:\left\{0,1, \ldots, m_{i}\right\} \rightarrow \mathbb{B}^{m_{i}}, \quad\left(b_{0}\right)_{i}(k)=(\underbrace{1,1, \ldots, 1}_{k}, 0, \ldots, 0) \text { for all } i \in I .
$$

Didier et al. [7] showed that Van Ham's embedding is essentially the only one satisfying nice properties which they call neighbour preservation and regulation preservation (see Remark 2 below). On the other hand, an apparent inconvenience of this method is that the resulting evolution function is defined only on the restricted domain $\operatorname{Im}(b)$, the set of admissible states [7]. In contrast, we will give a construction which produces a Boolean network defined on the whole state space $\mathbb{B}^{m_{1}+\cdots+m_{n}}$. The idea is to use a surjective map $\psi: \mathbb{B}^{m_{1}+\cdots+m_{n}} \rightarrow M$ rather than an embedding in the opposite direction. 
Remark 2. Properties similar to the first two in Definition 3 were introduced by Didier et al. [7] but only for embeddings $b: M \rightarrow \mathbb{B}^{l}$ (and mappings obtained by conjugation with embeddings Eq. (2)). Recall that an embedding $b: M \rightarrow \mathbb{B}^{l}$ is said to be neighbour-preserving if it satisfies $d\left(b(y), b\left(y^{\prime}\right)\right)=1$ for any $y, y^{\prime} \in M$ with $d\left(y, y^{\prime}\right)=1$. Also an embedding is said to be regulationpreserving if $G f_{b}\left(\mathbb{B}^{l}\right) \neq G f_{b}^{\prime}\left(\mathbb{B}^{l}\right)$ when $G f(M) \neq G f^{\prime}(M)$ for any $f, f^{\prime}: M \rightarrow M$; that is, the global interaction graphs of the Boolean networks obtained by conjugation differ when so do those of the multilevel networks. Our definitions are modified versions of theirs which apply to any mapping.

Here, we define a mapping from $\operatorname{Evo}(M)$ to $\operatorname{Evo}\left(\mathbb{B}^{l}\right)$ with $l=\left(m_{1}+\cdots+m_{n}\right)$, and a mapping from grid graphs over $M$ to grid graphs over $\mathbb{B}^{l}$, which possesses all three above properties.

Define a map $\psi: \mathbb{B}^{l} \rightarrow M$ by

$$
\psi\left(x_{1,1}, x_{1,2}, \ldots, x_{1, m_{1}}, x_{2,1}, \ldots, x_{n, m_{n}}\right)=\left(\left|y_{1}\right|, \ldots,\left|y_{n}\right|\right),
$$

where $y_{i}=\left(x_{i, 1}, \ldots, x_{i, m_{i}}\right) \in \mathbb{B}^{m_{i}}$ and $\left|y_{i}\right|=\sum_{k=1}^{m_{i}} x_{i, k}$. We denote the index set of $\mathbb{B}^{l}=\mathbb{B}^{m_{1}+\cdots+m_{n}}$ by $\boldsymbol{I}_{\mathbb{B}}=\left\{\left(i, j_{i}\right) \mid 1 \leq i \leq n, 1 \leq j_{i} \leq m_{i}\right\}$.

Definition 4. For an asymptotic multilevel evolution function $f \in \operatorname{Evo}(M)$, its binarisation $\mathbb{B}(f) \in$ $\operatorname{Evo}\left(\mathbb{B}^{l}\right)$ is defined by

$$
\mathbb{B}(f)_{i, j}(x):= \begin{cases}0 & \left(f_{i}(\psi(x))<\psi(x)_{i}\right) \\ x_{i, j} & \left(f_{i}(\psi(x))=\psi(x)_{i}\right) \\ 1 & \left(f_{i}(\psi(x))>\psi(x)_{i}\right) .\end{cases}
$$

Conversely, we have

$$
f_{i}(\psi(x))=\sum_{j=1}^{m_{i}} \mathbb{B}(f)_{i, j}(x) .
$$

The binarisation of a grid graph $\Gamma$ on $M$, denoted by $\mathbb{B}(\Gamma)$, is defined to be the grid graph on $\mathbb{B}^{l}$ such that there exists a directed edge $x \rightarrow x^{\prime}$ in $\mathbb{B}(\Gamma)$ if and only if $d\left(x, x^{\prime}\right)=1$ and there exists a directed edge $\psi(x) \rightarrow \psi\left(x^{\prime}\right)$ in $\Gamma$.

It is trivial to see $\mathbb{B}\left(f^{\Gamma}\right)=f^{\mathbb{B}(\Gamma)}$ and $\mathbb{B}(\Gamma(f))=\Gamma(\mathbb{B}(f))$. We now identify the image of binarisation $\operatorname{Evo}(M) \rightarrow \operatorname{Evo}\left(\mathbb{B}^{l}\right)$. The symmetric group $S_{m_{i}}$ acts on $\mathbb{B}^{m_{i}}$ by permuting the coordinates. We consider the coordinate-wise action of $\mathbb{S}=S_{m_{1}} \times S_{m_{2}} \times \cdots \times S_{m_{n}}$ on $\mathbb{B}^{l}=\mathbb{B}^{m_{1}} \times \mathbb{B}^{m_{2}} \times \cdots \mathbb{B}^{m_{n}}$. Since the map $\psi$ is invariant under this action, the binarisation $\mathbb{B}(f)$ has symmetry with respect to this action.

Definition 5. A Boolean network $f^{\prime}: \mathbb{B}^{l} \rightarrow \mathbb{B}^{l}$ is said to be $\mathbb{S}$-symmetric if

$$
f_{i, j}^{\prime}\left(x_{1,1}, \ldots, x_{n, m}\right)=f_{i, \sigma_{i}(j)}^{\prime}\left(x_{1, \sigma_{1}(1)}, x_{1, \sigma_{1}(2)}, \ldots, x_{1, \sigma_{1}\left(m_{1}\right)}, x_{2, \sigma_{2}(1)}, \ldots, x_{n, \sigma_{n}\left(m_{n}\right)}\right)
$$

for any $\sigma=\left(\sigma_{1}, \ldots, \sigma_{n}\right) \in \mathbb{S}$. Similarly, a Boolean grid graph $\Gamma^{\prime}$ is said to be $\mathbb{S}$-symmetric when an edge $x \rightarrow x^{\prime}$ exists if and only if so does $\sigma(x) \rightarrow \sigma\left(x^{\prime}\right)$.

For an $\mathbb{S}$-symmetric Boolean evolution function $f^{\prime}$ we obtain a well-defined evolution function $\psi \circ f^{\prime} \circ \psi^{-1}$. Similarly, for an $\mathbb{S}$-symmetric $\Gamma^{\prime}$, we obtain a grid graph over $M$ as the image under $\psi$.

Theorem 1. The binarisation induces a bijective mapping between the set $\operatorname{Evo}(M)$ of asymptotic evolution functions on $M$ and the set $\operatorname{Evo}\left(\mathbb{B}^{m_{1}+\cdots+m_{n}}\right)$ of Boolean evolution functions on $\mathbb{B}^{m_{1}+\cdots+m_{n}}$ which are $\mathbb{S}$-symmetric.

It immediately follows that Van Ham's embedding $b_{0}: M \rightarrow \mathbb{B}^{l}$ and our own map $\psi: \mathbb{B}^{l} \rightarrow M$ induce graph homomorphisms $\widetilde{b_{0}}: \Gamma(f) \rightarrow \Gamma(\mathbb{B}(f))$ and $\tilde{\psi}: \Gamma(\mathbb{B}(f)) \rightarrow \Gamma(f)$ for any $f \in \operatorname{Evo}(M)$ such that $\tilde{\psi} \circ \widetilde{b_{0}}$ is the identity. Thus, the binarisation is neighbour-preserving. We will see that it is also locally, and hence globally as well, regulation-preserving. We show that the dynamics of the system, namely, attractors in the state transition graph are preserved under binarisation.

In what follows, we often make use of the following two obvious facts:

\section{Lemma 1.}


- When there exists an edge $x \rightarrow x^{\prime}$ in $\mathbb{B}(\Gamma)$, there exists an edge $\psi(x) \rightarrow \psi\left(x^{\prime}\right)$ in $\Gamma$.

- When there exists an edge $y \rightarrow y^{\prime}$ in $\Gamma$, for any $x \in \psi^{-1}(y)$ there exists an edge $x \rightarrow x^{\prime}$ in $\mathbb{B}(\Gamma)$ for some $x^{\prime} \in \psi^{-1}\left(y^{\prime}\right)$.

Proposition 2. The strongly connected components of $\mathbb{B}(\Gamma)$ map surjectively onto those of $\Gamma$ via $\tilde{\psi}$. Moreover, attractors of $\mathbb{B}(\Gamma)$ map surjectively onto those of $\Gamma$ via $\tilde{\psi}$.

Proof. Assume that $x, x^{\prime} \in \mathbb{B}(\Gamma)$ are in the same strongly connected component. This means, there is a cycle containing $x, x^{\prime}$ and it maps to a cycle containing $\psi(x), \psi\left(x^{\prime}\right) \in \Gamma$. Therefore, $\psi(x), \psi\left(x^{\prime}\right)$ are in the same strongly connected component. Conversely, assume that there exists a cycle containing $y, y^{\prime} \in \Gamma$. For any vertex $x \in \psi^{-1}(y)$, there exists a vertex $x^{\prime} \in \psi^{-1}\left(y^{\prime}\right)$ and a cycle containing both $x$ and $x^{\prime}$. To sum up, the image of a strongly connected component of $\mathbb{B}(\Gamma)$ is a strongly connected component of $\Gamma$, and for any strongly connected component of $\Gamma$ there exists a strongly connected component of $\mathbb{B}(\Gamma)$ which maps to it. Since $\tilde{\psi}$ is a surjective graph homomorphism, attractors of $\mathbb{B}(\Gamma)$ map surjectively onto those of $\Gamma$ via $\tilde{\psi}$.

\section{Corollary 1.}

- A stable state exists in $\mathbb{B}(\Gamma)$ if and only if it does in $\Gamma$.

- A cyclic attractor exists in $\mathbb{B}(\Gamma)$ if and only if it does in $\Gamma$.

Proof. The first statement is trivial. For any cyclic attractor in $\Gamma$, there exists an attractor in $\mathbb{B}(\Gamma)$ which maps to it by the previous proposition. Since it contains more than one element, it is a cyclic attractor in $\mathbb{B}(\Gamma)$. Conversely, assume that there is a cyclic attractor in $\mathbb{B}(\Gamma)$. It contains at least two elements $x, x^{\prime}$ with $d\left(x, x^{\prime}\right)=1$. Their images $\psi(x), \psi\left(x^{\prime}\right)$ should be different since any two distinct elements in a single fibre (the inverse image of a point) $\psi^{-1}(\psi(x))$ have at least distance two. Thus, the image of the cyclic attractor contains at least two distinct elements $\psi(x), \psi\left(x^{\prime}\right)$ and is a cyclic attractor in $\Gamma$.

Theorem 2. The map $I_{\mathbb{B}} \rightarrow I$ defined by $\left(i, j_{i}\right) \mapsto i$ induces a surjective graph homomorphism on $G \mathbb{B}(f)(x) \rightarrow G f(y)$ for $y=\psi(x)$ and any $x \in \mathbb{B}^{l}$. More precisely, the following two statements hold.

(1) At any $y \in M$, if a positive (resp. negative) edge $i \rightarrow i^{\prime}$ exists in $G f(y)$, so does a positive (resp. negative) edge $(i, j) \rightarrow\left(i^{\prime}, j^{\prime}\right)$ in $G \mathbb{B}(f)(x)$ for some $j$ and $j^{\prime}$ at any $x \in \psi^{-1}(y)$.

(2) At any $x \in \mathbb{B}^{l}$, if a positive (resp. negative) edge $(i, j) \rightarrow\left(i^{\prime}, j^{\prime}\right)$ exists in $G \mathbb{B}(f)(x)$, so does a positive (resp. negative) edge $i \rightarrow i^{\prime}$ in $G f(\psi(x))$.

Proof. We only show the statements for the case of a positive edge, as the case of a negative edge follows by a similar argument.

For the first statement, assume that there exists a positive edge $i \rightarrow i^{\prime}$ in $G f(y)$. We have two cases $\partial_{i}^{+} f_{i^{\prime}}(y)>0$ and $\partial_{i}^{-} f_{i^{\prime}}(y)>0$. When $\partial_{i}^{-} f_{i^{\prime}}(y)=f_{i^{\prime}}(y)-f_{i^{\prime}}\left(y-e_{i}\right)>0$, for any $x \in \psi^{-1}(y)$ there exists $j$ such that $x_{i, j}=1$ since $y_{i}>0$. By Eq. (3) there must exist some $j^{\prime}$ such that $\mathbb{B}(f)_{i^{\prime}, j^{\prime}}(x)-\mathbb{B}(f)_{i^{\prime}, j^{\prime}}\left(x-e_{i, j}\right)=1$. This means there exists a positive edge $(i, j) \rightarrow\left(i^{\prime}, j^{\prime}\right)$ in $G \mathbb{B}(f)(x)$. A similar argument applies when $\partial_{i}^{+} f_{i^{\prime}}(y)>0$.

For the second statement, assume that there exists a positive edge $(i, j) \rightarrow\left(i^{\prime}, j^{\prime}\right)$ in $G \mathbb{B}(f)(x)$. When $x_{i, j}=0$, this means $\mathbb{B}(f)_{i^{\prime}, j^{\prime}}(x)=0$ and $\mathbb{B}(f)_{i^{\prime}, j^{\prime}}\left(x+e_{i, j}\right)=1$. Since $\psi(x)+e_{i}=$ $\psi\left(x+e_{i, j}\right)$, we have $f_{i^{\prime}}\left(\psi(x)+e_{i}\right)-f_{i^{\prime}}(\psi(x))=\sum_{k=1}^{m_{i^{\prime}}}\left(\mathbb{B}(f)_{i^{\prime}, k}\left(x+e_{i, j}\right)-\mathbb{B}(f)_{i^{\prime}, k}(x)\right)$. Since $\mathbb{B}(f)_{i^{\prime}, k}\left(x+e_{i, j}\right)-\mathbb{B}(f)_{i^{\prime}, k}(x) \geq 0$ for all $k$ and $\mathbb{B}(f)_{i^{\prime}, j^{\prime}}\left(x+e_{i, j}\right)-\mathbb{B}(f)_{i^{\prime}, j^{\prime}}(x)=1$, we have $f_{i^{\prime}}\left(\psi(x)+e_{i}\right)-f_{i^{\prime}}(\psi(x))>0$. This in turn means that there exists a positive edge $i \rightarrow i^{\prime}$ in $G f(\psi(x))$. A similar argument applies to the case when $x_{i, j}=1$.

Intuitively speaking, (1) says all the regulation in the original multilevel network is captured in the converted Boolean network, while (2) says all the regulation in the converted network comes from the original multilevel network.

Corollary 2. An asymptotic evolution function $f$ over $M$ has a positive (negative) type-1 functional circuit if so does its binarisation $\mathbb{B}(f)$. 
Note that two negative arrows $(i, j) \rightarrow\left(i, j^{\prime}\right)$ and $\left(i, j^{\prime}\right) \rightarrow(i, j)$ in $G \mathbb{B}(f)(x)$, both of which correspond to a negative self-loop $i \rightarrow i$ in $G \mathbb{B}(f)(x)$, can be composed to produce a positive circuit. This positive functional type- 1 circuit corresponds the one which is the composition of a single negative self-circuit with itself in $G f(\psi(x))$.

Example 4. We give two characteristic examples of the binarisation.

Consider the evolution function $f(y)=2-y$ over $M=\{0,1,2\}$. Its binarisation is

$$
\mathbb{B}(f)(x)= \begin{cases}(1,1) & (x=(0,0)) \\ x & (x=(1,0),(0,1)) \\ (0,0) & (x=(1,1))\end{cases}
$$

The corresponding state transition graphs are
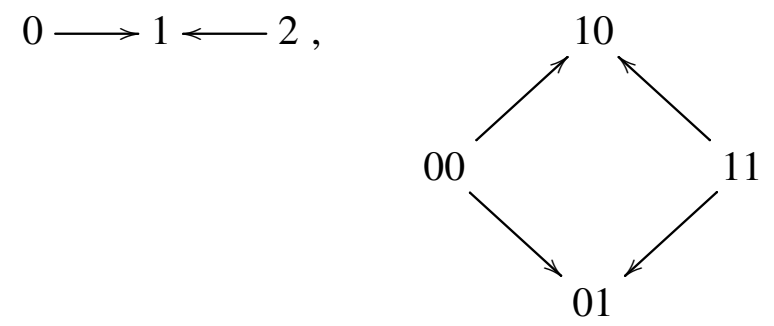

The interaction graphs at $y=0$ and $x=(0,0)$ respectively look:

$$
G f(0)=\stackrel{-}{y}, \quad G \mathbb{B}(f)(0,0)=x_{11} \overbrace{\tau_{-}} x_{12}
$$

Notice that the self-loop on $y_{1}$ corresponds to each of the edges $x_{11} \rightarrow x_{12}$ and $x_{11} \leftarrow x_{12}$. In particular, the converse to Corollary 2 does not hold.

Consider another evolution function over $M=\{0,1,2\}$ defined by $f(y)=\left\{\begin{array}{ll}y & (y=0,1) \\ 0 & (y=2) .\end{array}\right.$ Its binarisation is $\mathbb{B}(f)(x)=\left\{\begin{array}{ll}x & (x \neq(1,1)) \\ (0,0) & (x=(1,1)) .\end{array}\right.$ The corresponding state transition graphs are

$$
0---1 \longleftarrow 2 \text {, }
$$

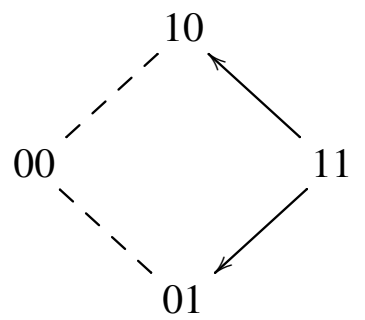

The interaction graphs at $y=1$ and $x=(0,1)$ respectively look:

$$
G f(1)=\overbrace{y_{+}^{2},}^{-} G \mathbb{B}(f)(0,1)=x_{11} \overbrace{12})+
$$

2.3. Another extension method. Recently, Tonello [16] has independently constructed a mapping which also extends Van Ham's while preserving the dynamics and the local regulations in a more stringent sense than ours. Her method was used to produce a counter-example to Conjecture 1 as well. Her mapping can be described in our context as follows:

$$
f \mapsto b_{0} \circ f \circ \psi,
$$

where $f$ is a stepwise function. Compared to ours, her method yields fewer arrows in the state transition graph. Her strategy was to stipulate the converted function to take values in the admissible 
region $\operatorname{Im}\left(b_{0}\right)$, whereas ours was to equip the converted function with the symmetry described in Theorem 1 .

\section{Results}

3.1. Lambda phage. As an illustration, we first apply our method to the 2-variable lambda phage model proposed by Thieffry and Thomas [17].

The lambda phage is a bacterial virus that infects $E$. coli. It is a temperate phage, i.e. it can either multiply and eventually kill the host cell (lytic phase), or integrate its DNA into the bacterial chromosome (lysogenic phase), conferring the cell immunity against super-infection by other lambda phage. The switch between lysis and lysogeny, as modelled by Thieffry and Thomas, is essentially controlled by a positive feedback circuit between genes $c I$ and cro. The two genes inhibit each other, such that $c I$ dominates the lysogenic phase, whereas cro is active during the lytic phase. The gene cro further inhibits its own activity. The system is modelled by a discrete system with the state space $M=\{(c I, c r o) \in\{0,1\} \times\{0,1,2\}\}$. The dynamics displays a stable state with high $c I$ and low cro activity, and a two-state cyclic attractor with low $c I$, and cro oscillating around its activity threshold (Fig. 1, left), which the authors describe as homeostasis.
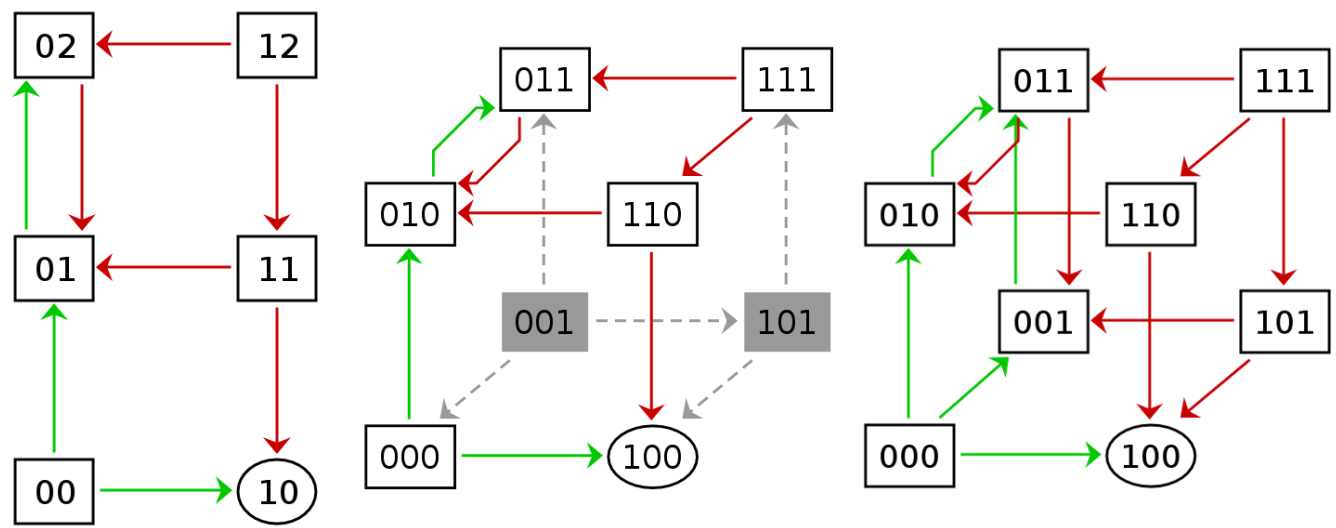

FIGURE 1. State transition graphs for the lambda phage model. Left, the original, multilevel model [17]; centre, the Boolean version obtained using Van Ham's method; right, the Boolean version obtained using our method.

Table 1 shows how the same dynamics can be encoded using a stepwise or asymptotic evolution function. Notice that the stepwise function creates a positive feedback on $\operatorname{cro}\left(\hat{f}_{\text {cro }}(0,1)-\hat{f}_{\text {cro }}(0,0)>0\right.$, and $\left.\hat{f}_{c r o}(1,2)-\hat{f}_{c r o}(1,1)>0\right)$ that is not visible in the asymptotic function. This difference in the global regulatory graphs is shown in Fig. 2 .

TABLE 1. Truth table of the lambda phage model

\begin{tabular}{cc|cc|cc}
$c I$ & cro & $\hat{f}_{c I}$ & $\hat{f}_{\text {cro }}$ & $\bar{f}_{c I}$ & $\bar{f}_{c r o}$ \\
\hline 0 & 0 & 1 & 1 & 1 & 2 \\
0 & 1 & 0 & 2 & 0 & 2 \\
0 & 2 & 0 & 1 & 0 & 0 \\
1 & 0 & 1 & 0 & 1 & 0 \\
1 & 1 & 0 & 0 & 0 & 0 \\
1 & 2 & 0 & 1 & 0 & 0
\end{tabular}

Table gives the target level of $c I$ and $c r o$ in each state, encoded using either the stepwise or asymptotic evolution function.

Boolean systems are generated by Van Ham's method and ours with the state space $\{(c I$, cro 1, cro 2$) \in$ $\left.\mathbb{B}^{3}\right\}$. However, since Van Ham's method yields a dynamics with as many states as the original, multilevel dynamics (Fig. 1, centre), the system thus obtained includes a "non-admissible" region (grey 
area in the Figure) whose states do not have any counterpart in the multilevel model. To make comparison, we extended the domain of the Boolean model obtained by Van Ham's method (Fig. 11, centre) by completing the grey dashed arrows in such a way that

- it does not create any extra arrows in the global interaction graph that is not already visible elsewhere within the admissible region

- and there is no outgoing arrow in the state transition graph from an admissible state to a non-admissible state.

This extension is based on our understanding of Van Ham's original publication [6]. The corresponding global regulatory graph is shown in Fig. 2 (centre).
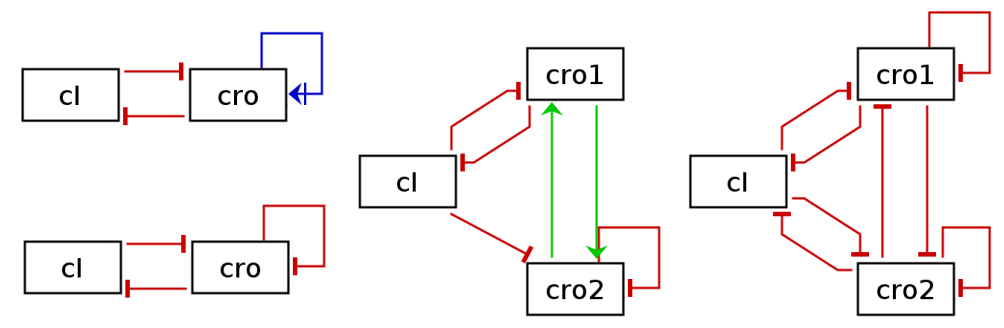

FIGURE 2. Global interaction graphs for the lambda phage model. Left, the original, multilevel model [17]: top, stepwise evolution function, bottom, asymptotic evolution function; centre, the Boolean version obtained using Van Ham's method; right, the Boolean version obtained using our method.

In contrast, the dynamics produced by our method is more complex, and it occupies the whole state space: every state has a counterpart in the original multilevel model. However, the two-state attractor of the original model is now represented by a three-state attractor, where state 011 corresponds to state 02 and both states 001 and 010 correspond to state 01 . Another difference is that, in the model obtained with Van Ham's method, variables cro 1 and cro 2 are ordered and represent levels 1 and 2, respectively, of the original multilevel cro. In the Boolean model generated with our method, cro 1 and cro 2 are interchangeable and equivalent: whether one represents level 1 or 2 depends on the other, and thus, on the context of each particular state.

Finally, an important difference appears at the local level (Fig. 3). Using Van Ham's method, local graphs may include edges that have no visible counterpart in the local graph of the corresponding multilevel state. For example, while in 02 the only visible regulation is the negative loop on cro, Van Ham's method adds an edge from $c r o 1$ to $c I$ in 011, whereas the corresponding local graph obtained using our method includes only regulations between cro 1 and cro2. Similarly, in 10, the only visible edges occur between $c I$ and cro, and the same is true in 100 using our method; however, using Van Ham's method an additional edge between cro1 and cro2 becomes visible. Our method generates an "extra" positive circuit between cro1 and cro2, which in multilevel model corresponds to the composition of the negative self circuit on cro with itself. Such positive circuits can only appear between variables that represent the same multilevel variable (see Corollary 2).

3.2. Boolean counter-example to Conjecture 1. One of the main goals in genetic regulatory network analysis is to find relations between circuits in the interaction graphs and attractors in the state transition graphs. Here we list a few known results in this direction:

(1) If $f$ has no type-1 functional circuit, $\Gamma(f)$ has a unique stable state $x([18])$.

(2) If $f$ has no type-1 functional positive circuit, $\Gamma(f)$ has a unique attractor $([11,10])$.

(3) If there exists a cyclic attractor in $\Gamma(f), G f(M)$ must contain a negative circuit ([12]).

Notice that the first two statements connect the asymptotic behaviour of the dynamics with the local interaction graph at some $x \in M$, while the last one does so with the global interaction graph $G f(M)$. This naturally gives rise to the following conjectures:

Conjecture 1 ([9, Question 1],[12, Question 1,2]). 


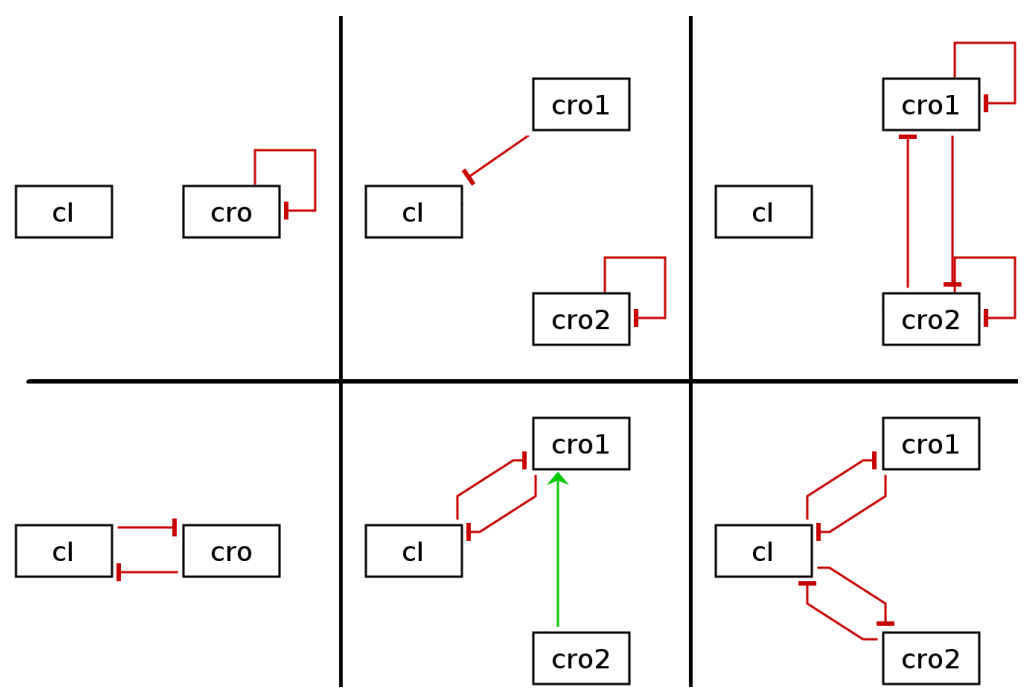

FIGURE 3. Local interaction graphs for the lambda phage model. Left, the original, multilevel model [17]; centre, the Boolean version obtained using Van Ham's method; right, the Boolean version obtained using our method. Top, local graphs for low $c I$ and high cro (states 02 or 011); bottom, local graphs for states with high $c I$ and low cro (states 10 and 100). Local graphs in 02 and 10 are identical under the stepwise or asymptotic evolution function.

(1) If $f$ has no type-1 functional negative circuit, then $\Gamma(f)$ has no cyclic attractors.

(2) If $f$ has no type-1 functional negative circuit, then $\Gamma(f)$ has a stable state.

Note that the second statement is weaker in the sense that it follows from the first one.

Richard, together with Comet [12, Example 6], gave a counter-example to the conjectures when $M=\{0,1,2,3\}^{2}$. The grid graph $\Gamma$ over $M=\{0,1,2,3\}^{2}$ given in [12, Example 6] has no stable state and there exists no type-1 negative functional circuit in the interaction graph of $\bar{f}^{\Gamma}$ (see Fig. 4). The existence of Boolean counter-examples was left as an open problem in [12].

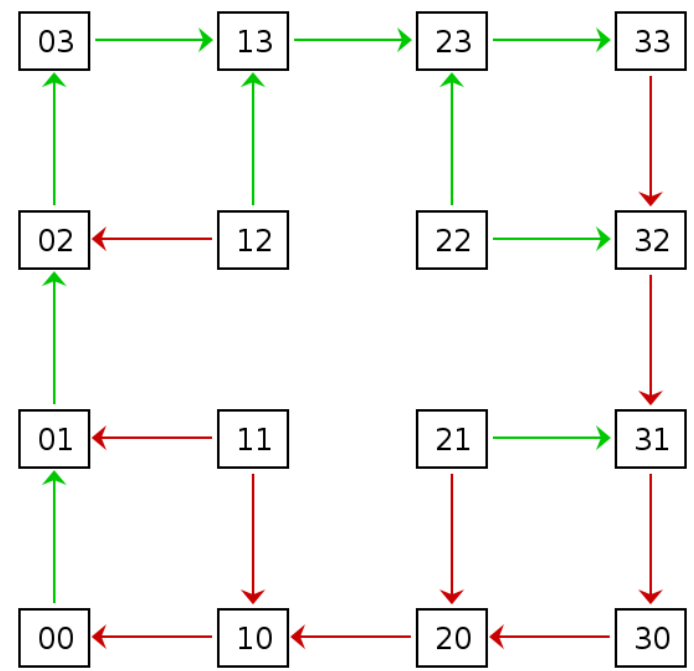

Figure 4. A multilevel counter-example given in [12]

By Corollary 2, the Boolean grid graph $\mathbb{B}(\Gamma)$ yielded by our method (Fig. 5) has no type-1 negative functional circuit in the interaction graph of the binarisation $\mathbb{B}\left(\bar{f}^{\Gamma}\right)$. Furthermore, by Corollary 1 there is no stable state in the state transition graph of $\mathbb{B}\left(f^{\top}\right)$. Thus, we obtain a new Boolean counterexample to Conjecture 1 .

Finally, we note that Ruet recently gave a systematic way to produce counter-examples to the conjectures for the Boolean case [13]. Our method is very different from Ruet's and while his example 


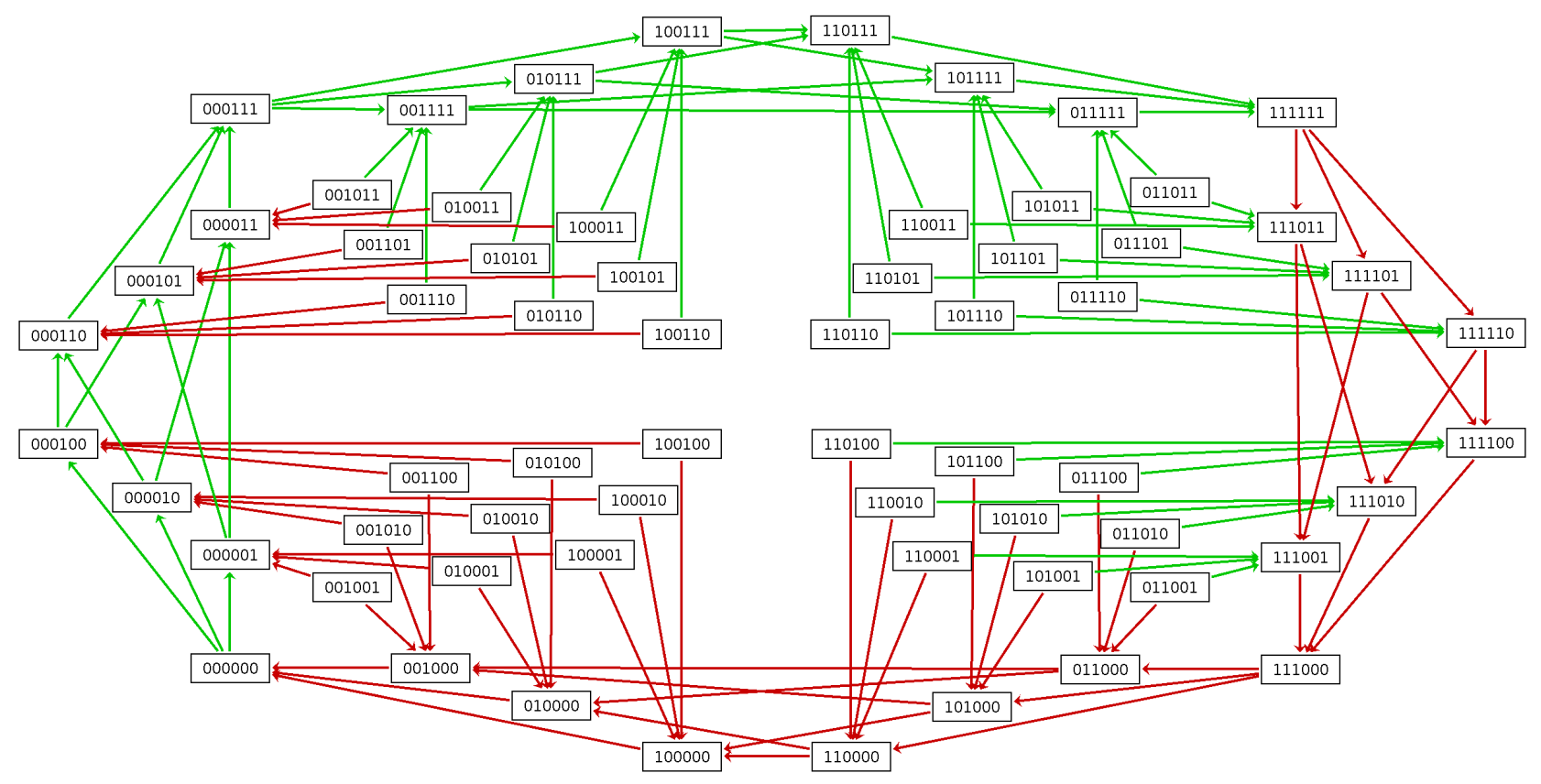

Figure 5. A Boolean counter-example to Conjecture 1 The binarisation of the multilevel counter-examples gives a Boolean counter-example

possesses a special property that it has an attractive cycle, our counter-example has a smaller dimension of 6. For comparison, we include an example based on Ruet's theory [13] Fig. 6.

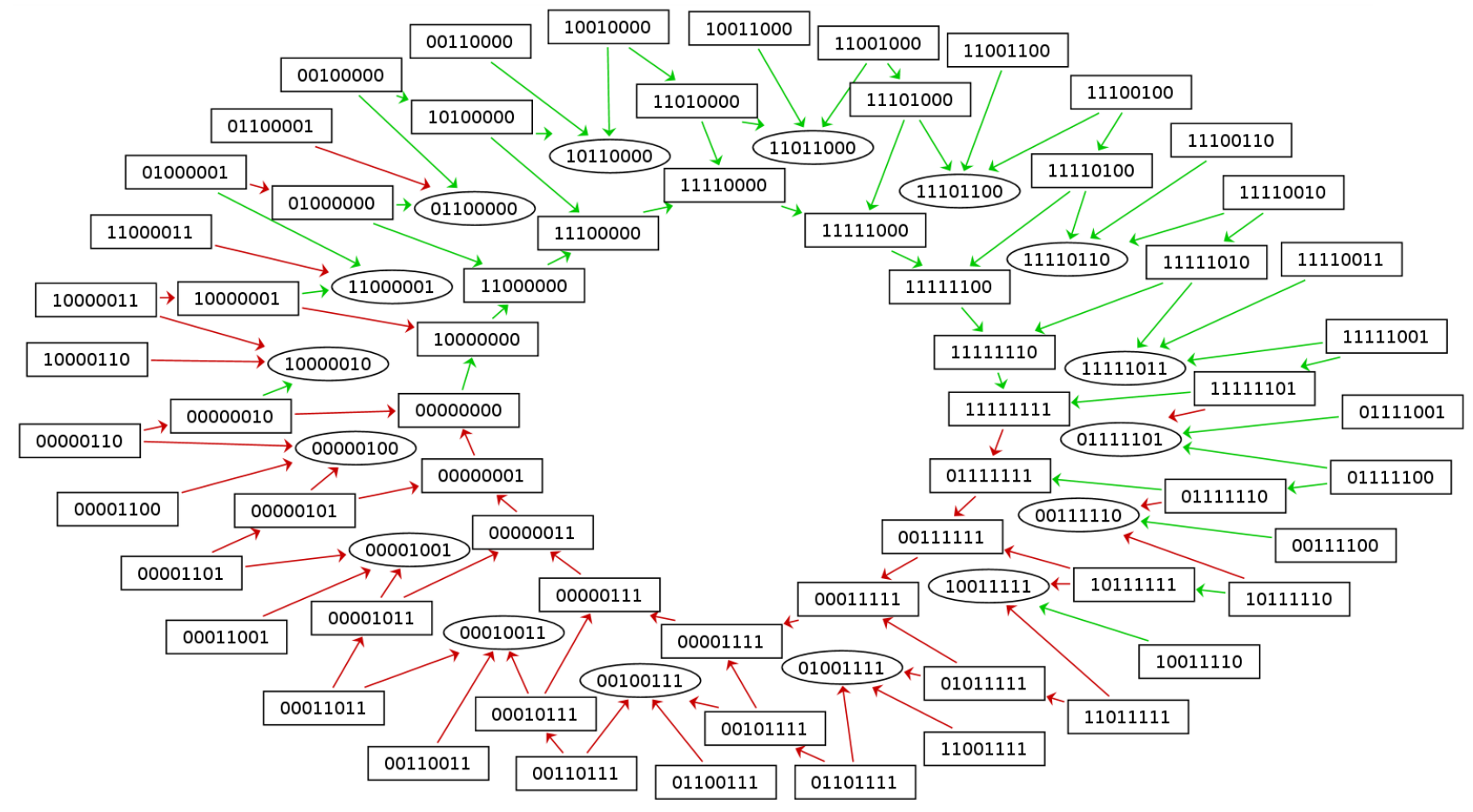

FIGURE 6. Antipodal attractive cycle with no negative circuit. States not shown are all stable states. Adapted from [13].

3.3. Implementation. We implemented our method in the form of a Perl script. For comparison, Tonello's method [16] is also implemented in the same script. It is available at [19] under the MIT license. The input is a multilevel evolution function described in the Truth table format [20] and the output is a Boolean evolution function described in the same format. The maximum levels $m_{i}$ for each 
gene is automatically detected. When the input function is defined on $\left\{0,1, \ldots, m_{1}\right\} \times\left\{0,1, \ldots, m_{2}\right\} \times$ $\cdots \times\left\{0,1, \ldots, m_{n}\right\}$, the converted Boolean network is defined on $\mathbb{B}^{m_{1}+m_{2}+\cdots+m_{n}}$.

\section{Discussion AND CONCLUSIONS}

In the discrete formulation of gene regulatory networks, a system is commonly modelled by a function. When some genes take more than two levels, there are multiple choices for functions having the same (asynchronous) state transition graph. We single out a unique choice, which we call the asymptotic evolution function (Proposition 1). Then, we introduced a mapping which converts an asymptotic evolution function to a Boolean evolution function (Definition 4). This mapping preserves dynamical and regulatory properties (Proposition 2, Theorem 2), thus allowing us to analyse multilevel networks by methods developed for Boolean networks.

Mappings from multilevel to Boolean networks have been used in the study of gene regulatory networks. In particular, Van Ham's mapping has been shown by Didier et al. to be essentially the only method to provide a one-to-one, neighbour-preserving and regulation-preserving Boolean representation of multilevel models [7]. However, although the authors did suggest that the mapping could be useful to study the role of regulatory circuits, the question of how interaction functionality contexts are preserved had not been studied so far.

One such instance is Thomas's conjecture, which states that the existence of a cyclic attractor in the asynchronous state transition graph requires that of local negative circuit. The conjecture has recently been given a Boolean counter-example by P. Ruet [13]. Until then, although A. Richard and and J-P. Comet had produced a multilevel counter-example [12], the Boolean case remained open. It is straightforward to apply Van Ham's method to the counter-example in order to obtain a Boolean model, but it is defined only on the admissible region. To extend the model to the whole Boolean state space, while preserving its dynamics and regulatory relation, is highly non-trivial. The method we propose has been designed specifically to circumvent the problem. The idea was to avoid extra interactions and circuits by extending the state transition graph obtained with Van Ham's method in such a way that we have "parallel trajectories" going through the whole state space. This was achieved by loosening the one-to-one criterion such that states including intermediate values in the multilevel model would match with several states in the Boolean version, effectively creating equivalent Boolean transitions for each multilevel transition in the original model.

In a sense, our method works opposite to Van Ham's: instead of embedding multilevel states into Boolean states, we define a Boolean model such that each Boolean state can be mapped to a multilevel state. With our method, interaction functionality is preserved, and thus all local interaction graphs in the Boolean model come from their counterpart in the original, multilevel model. In contrast, Van Ham's method only preserves the global interaction graph.

One limitation of our method is that the synchronous dynamics of the original multilevel model can not be directly retrieved from the Boolean model produced by the conversion. Here, by synchronous dynamics we mean the state transition graph having edges $x \rightarrow f(x)$ instead of (1) (see, for example, [21]). Synchronous state transition is often deemed unrealistic since it assumes all processes are realised simultaneously with the same delay (see discussion by Abou-Jaoudé et al. [1]). Nevertheless, the synchronous mode is still a popular update method in simulation due to its simplicity, and occasionally used for multilevel models (see e.g. Chifman et al. [22] for a recent example). If our binarisation is used for a synchronous simulation, any increase in a variable would translated into its increase to the maximum value. It is worth noting that the counter-examples for Conjecture 1 considered in $\$ 3.2$ (Richard-Comet's one and its binarisation) have no stable state in the synchronous state transition graph as well (and have no type-1 negative functional circuit in the interaction graph).

Finally, our results highlight two opposite strategies, stepwise and asymptotic, for writing the evolution function of a multilevel model. While both suppress inter-genes regulations, the stepwise tends to add positive self-regulations, whereas the asymptotic tends to add negative self-regulations. This work contributes to a better understanding of the different ways to represent a multilevel system, for different ways can represent the same model [15], which causes ambiguities in the notation. 


\section{ACKNOWLEDGEMENTS}

The authors are grateful to Paul Ruet for explaining his result in [13], to Elisa Tonello for fruitful discussion and careful reading of our draft, and to Yuki Ikawa and Sergey Tishchenko for their help in the early stage of this work. The second named author is partially supported by JST PRESTO Grant Number JPMJPR16E3, Japan.

\section{REFERENCES}

[1] Abou-Jaoudé, W., Traynard, P., Monteiro, P.T., Saez-Rodriguez, J., Helikar, T., Thieffry, D., Chaouiya, C.: Logical modeling and dynamical analysis of cellular networks. Front Genet 7, 94 (2016). doi:10.3389/fgene.2016. 00094

[2] Stoll, G., Viara, E., Barillot, E., Calzone, L.: Continuous time boolean modeling for biological signaling: application of gillespie algorithm. BMC Syst Biol 6, 116 (2012). doi 10.1186/1752-0509-6-116

[3] MacNamara, A., Terfve, C., Henriques, D., Peñalver Bernabé, B., Saez-Rodriguez, J.: State-time spectrum of signal transduction logic models. Physical Biology 9(4), 045003 (2012)

[4] Helikar, T., Kowal, B., McClenathan, S., Bruckner, M., Rowley, T., Madrahimov, A., Wicks, B., Shrestha, M., Limbu, K., Rogers, J.A.: The cell collective: toward an open and collaborative approach to systems biology. BMC Syst Biol 6, 96 (2012). doi 10.1186/1752-0509-6-96

[5] Remy, É., Ruet, P., Thieffry, D.: Positive or negative regulatory circuit inference from multilevel dynamics. Lecture Notes in Control and Information Sciences 341, 263-70 (2006)

[6] Van Ham, P.: Kinetic logic: A boolean approach to the analysis of complex regulatory systems. Lecture notes in Biomathematics., vol. 29, pp. 326-43. Levin, S. (1979). Chap. How to deal with variables with more than two levels

[7] Didier, G., Remy, E., Chaouiya, C.: Mapping multivalued onto boolean dynamics. J. Theor. Biol. 270(1), 177-84 (2011). doi $10.1016 / \mathrm{j} . j \mathrm{tbi} .2010 .09 .017$

[8] Thomas, R.: On the relation between the logical structure of systems and their ability to generate multiple steady states or sustained oscillations. In Springer series in synergies: Numerical methods in the study of critical phenomena, vol. 9, pp. 180-193. Della Dora, Jean and Demongeot, Jacques and Lacolle, Bernard (1981). doi $10.1007 / 978-3-642-81703-8 \_24$

[9] Comet, J.-P., Noual, M., Richard, A., Aracena, J., Calzone, L., Demongeot, J., Kaufman, M., Naldi, A., Snoussi, E.H., Thieffry, D.: On circuit functionality in boolean networks. Bull. Math. Biol. 75(6), 906-19 (2013). doi 10 . 1007/s11538-013-9829-2

[10] Remy, É., Ruet, P., Thieffry, D.: Graphic requirements for multistability and attractive cycles in a boolean dynamical framework. Advances in Applied Mathematics 41,335-350 (2008). doi:10.1016/j . aam.2007.11.003

[11] Richard, A., Comet, J.-P.: Necessary conditions for multistationarity in discrete dynamical systems. Discrete Applied Mathematics 155, 2403-13 (2007). doi 10.1016/j.dam.2007.04.019

[12] Richard, A.: Negative circuits and sustained oscillations in asynchronous automata networks. Adv. Appl. Math. 44(4), 378-392 (2010). doi $10.1016 /$ j . aam.2009.11.011

[13] Ruet, P.: Negative local feedbacks in boolean networks. Discrete Applied Mathematics 221, 1-17 (2017). doi:10. $1016 / j$.dam.2017.01.001

[14] Thomas, R., D’Ari, R.: Biological Feedback. CRC Press, Inc., 2000 Corporate Blvd, N.W., Boca raton, Florida, 33431, United States of America (1990). http://hal.archives-ouvertes.fr/hal-00087681/en/

[15] Streck, A., Lorenz, T., Siebert, H.: Minimization and equivalence in multi-valued logical models of regulatory networks. Natural Computing 14, 555-566 (2015). doi:10.1007/s11047-015-9525-2

[16] Tonello, E.: On the conversion of multivalued gene regulatory networks to Boolean dynamics. ArXiv e-prints (2017). 1703.06746

[17] Thieffry, D., Thomas, R.: Dynamical behaviour of biological regulatory networks-ii. immunity control in bacteriophage lambda. Bull. Math. Biol. 57(2), 277-97 (1995). doi:10.1016/0092-8240(94)00037-D

[18] Shih, M.-H., Dong, J.-L.: A combinatorial analogue of the jacobian problem in automata networks. Advances in Applied Mathematics 34(1), 30-46 (2005). doi:10.1016/j . aam.2004.06.002

[19] Kaji, S.: A multilevel to Boolean gene regulatory network converter. GitHub (2017)

[20] Naldi, A., Berenguier, D., Fauré, A., Lopez, F., Thieffry, D., Chaouiya, C.: Logical modelling of regulatory networks with ginsim 2.3. BioSystems 97(2), 134-9 (2009). doi 10.1016/j. biosystems.2009.04.008

[21] Remy, E., Mossé, B., Chaouiya, C., Thieffry, D.: A description of dynamical graphs associated to elementary regulatory circuits. Bioinformatics 19 Suppl 2, 172-8 (2003). doi:10.1093/bioinformatics/btg1075

[22] Chifman, J., Arat, S., Deng, Z., Lemler, E., Pino, J.C., Harris, L.A., Kochen, M.A., Lopez, C.F., Akman, S.A., Torti, F.M., Torti, S.V., Laubenbacher, R.: Activated oncogenic pathway modifies iron network in breast epithelial cells: A dynamic modeling perspective. PLoS Comput. Biol. 13(2), 1005352 (2017). doi:10.1371/journal .pcbi. 1005352

(A. Fauré) Department of Physics and Information Science 
YAMAGUCHI UNIVERSITY

1677-1, Yoshida, Yamaguchi 753-8512, JapaN

E-mail address: afaure@yamaguchi-u.ac.jp

(S. Kaji) Department of Mathematics

YAMAGUCHI UNIVERSITY

1677-1, YoshIDA, YAMaguchI 753-8512, JAPAN

/ JST PRESTO

E-mail address: skaji@yamaguchi-u.ac.jp 